\title{
The potential role of nontyphoidal salmonellosis in gastric cancer: a nationwide matched cohort study
}

\author{
Renin Chang ${ }^{1,2,3} \cdot$ James Cheng-Chung Wei ${ }^{4,5,6} \cdot$ Mei-Chen Lin ${ }^{7} \cdot$ Yao-Min Hung $8,9,10$
}

Received: 19 July 2020 / Accepted: 28 September 2020 / Published online: 1 November 2020

(c) The International Gastric Cancer Association and The Japanese Gastric Cancer Association 2020

\begin{abstract}
Background The research is to explore the association between nontyphoidal salmonellosis (NTS) and subsequent gastric cancer.

Methods We conducted a retrospective study by analyzing hospitalization dataset from the National Health Insurance Research Database in Taiwan. Patients aged 20 years and older with NTS $(n=9097)$ admitted between January 1, 2000, and December 31, 2012, were enrolled and followed up until December 31, 2013. The primary outcome was the incidence of gastric cancer. Cox proportional hazards regression was used to estimate the risk of malignancy, accounting for the competing risk of death. In addition, we conducted a sensitivity analysis by propensity score matching and exclusion of malignancy within 1 year observation to minimize measurable confounding and protopathic bias. Negative controls were applied to examine the presence of possible unmeasured confounders in the study.

Results The study included 18194 patients (9097 in each NTS and non-NTS group). The median follow-up time was 7 years. The incidence density rate of gastric cancer was 0.72 per 1000 person-years for the NTS group and 0.40 per 1000 personyears for the non-NTS group. The NTS group had a modestly higher risk of gastric cancer (aHR, 2.02; 95\% CI 1.18-3.45) than the non-NTS group. The sensitivity analyses revealed consistent results.

Conclusions Patients with NTS are associated with increased risk of subsequent gastric cancer compared with non-NTS patients. Future research is needed to examine whether NTS is parallel, reactive or causative to gastric cancer.
\end{abstract}

Keywords Nontyphoidal salmonellosis · Gastric cancer · Cohort

Electronic supplementary material The online version of this article (https://doi.org/10.1007/s10120-020-01132-x) contains supplementary material, which is available to authorized users.

Yao-Min Hung

ymhung1@gmail.com

Renin Chang

rhapsody1881@gmail.com

James Cheng-Chung Wei

wei3228@gmail.com

Mei-Chen Lin

coolindm@gmail.com

1 Department of Emergency Medicine, Kaohsiung Veterans General Hospital, Kaohsiung, Taiwan

2 Department of Recreation Sports Management, Tajen University, Pingtung, Taiwan

3 Institute of Biotechnology and Chemical Engineering, I-Shou University, Kaohsiung, Taiwan

4 Division of Allergy, Immunology and Rheumatology, Chung Shan Medical University Hospital, Taichung, Taiwan
5 Institute of Medicine, Chung Shan Medical University, Taichung 40203, Taiwan

6 Graduate Institute of Integrated Medicine, China Medical University, Taichung, Taiwan

7 Management Office for Health Data, China Medical University Hospital, Taichung 40447, Taiwan

8 Department of Internal Medicine, Kaohsiung Municipal United Hospital, 976, Jhong-hua 1st Rd, Gu-shan Dist., Kaohsiung 80457, Taiwan

9 Institute of Medicine, Chung Shan Medical University, Taichung, Taiwan

10 Shu-Zen Junior College of Medicine and Management, Kaohsiung, Taiwan 


\section{Introduction}

Cancer is a well-known adversary of public health, with both the disease and humans striving to survive. Despite the many efforts devoted to the study of cancer, it remains the leading cause of death in human beings. Indeed, cancer cells originate from our normal cells by multi-factorial carcinogenesis, including chronic infections and inflammation [1,2]. Since the beginning of the twentieth century, infection has emerged as a fundamental aspect of cancer causation, with a growing number of pathogens recognized as oncogenic [3]. Bacteria may become involved in cancer cell formation by causing inflammation, releasing toxins and metabolites, and reprogramming host cell signaling pathways during their life cycle [4]. Identification of such infectious pathogens is an important and effective way to break this potential chain of oncogenesis and to prevent normal cells from transforming to cancer cells; thus, it constitutes a priority for cancer prevention [3].

To date, many bacteria have been regarded as risk factors for cancer [5-8]. Periodontal disease related pathogens are regarded as having potential roles in the development of pancreatic cancer [9-11]. In recent studies, nontyphoidal salmonellosis (NTS) was found to be associated with colon cancer $[12,13]$. The stomach plays an important role in human immunity and destroys swallowed or ganisms, but, besides Helicobacter pylori (H. pylori), is it possible that another acidtolerant pathogen a potential carcinogen. The incidence rate of salmonellosis exceeds $1,000,000$ cases per year $(15,600$ hospitalizations; 550 deaths) [14] in the US. NTS can resist the acid environment in the stomach and colonize the small and large intestine [15]. Infection occurs after the ingestion of $10^{5}-10^{6}$ bacteria. Some studies have shown that salmonellae can resist the acidity of gastric juice and the interior environment of macrophages at a $\mathrm{pH}$ level of about 5-6 and become longer-staying persisters with heterogeneous phenotypes [16]. Furthermore, interest in gut microbiota and homeostasis has become widespread in recent years for their complex roles in many kinds of major catastrophic illnesses, such as autoimmune disease, stroke, and tumor formation [17].

Because the influence of NTS on the alimentary tract and its role in cancer are issues of debate, we conducted a populationbased longitudinal cohort study to provide an epidemiological evidence regarding the potential role of nontyphoid Salmonella infection in subsequent development of gastric cancer.

\section{Materials and methods}

\section{Data sources}

This study was conducted using data from the National Health Insurance Research Database (NHIRD), including the claims data from Taiwan's National Health Insurance (NHI) program, which was established in 1995 and covers over $99.99 \%$ of Taiwan's 23 million citizens. Insurance benefits include inpatient, ambulatory, emergency, dental, and traditional Chinese medicine services. Quarterly expert reviews on random samples of claims data, with a sampling rate of 1 in 50-100, are performed by the Bureau of NHI to ensure accuracy. The NHI records diseases on the basis of the International Classification of Diseases, Ninth Revision, Clinical Modification (ICD-9-CM). Before releasing the database for research, the original identification numbers were anonymized to protect patients' privacy.

The NHI Registry for Catastrophic Illness provided comprehensive utilization and enrollment information for all patients with severe diseases under the NHI program. All cancers were included in the category of catastrophic illness. Patients with Catastrophic Illness Certificates (CICs) are exempt from co-payments related to treatment and follow-up for the specific disease. The applications of CICs are reviewed by the Bureau of NHI. For the approval of CICs for cancer, histological confirmation of malignancy and associated laboratory and imaging studies are subjected to mandatory peer review. Therefore, the records of CICs are reliable.

\section{Study design}

\section{Setting}

This was a population-based retrospective matched-cohort study using NHIRD in Taiwan. To determine the association between NTS and gastric cancer, we compared the incidence of gastric cancer in two matched groups. The study was approved by the Research Ethics Committee of China Medical University and Hospital in Taiwan approved the study (CMUH-104-REC2-115-(AR4)). The requirement of informed consent was waived, because data were collected from the NHIRD. The study followed the "Strengthening the Reporting of Observational Studies in Epidemiology" reporting guideline.

\section{Participants and matching}

All adults newly diagnosed with NTS (International Classification of Diseases, Ninth Revision, Clinical Modification [ICD-9-CM] codes 003.**) admitted between January 1, 2000, and December 31, 2012, were enrolled and followed up until December 31, 2013. We defined the index date as the first hospitalized NTS diagnosis date, and this date was assigned to the respective matched non-NTS-exposed individuals. Doctors responsible for the patient are required to make diagnoses with the appropriate ICD code according to comprehensive evaluation and examination, including the analysis of stool and/or blood culture. The coding system 
is deemed valid, because the government regularly audits claims for the purpose of payment. Fines for fraud are 100 times the amount of the false claims charged to the National Health Insurance Bureau. People with a history of NTS or gastrointestinal cancer before the index date or having gastrointestinal cancer after the index date within 6 months were excluded, as were those with incomplete age or sex information. To minimize surveillance bias, the non-NTS group was drawn through random computerized sampling from the same hospitalization dataset, which consisted of patients who had never been diagnosed with NTS. Both groups were frequency-matched at a ratio of $1: 1$ by age (with a unit of 5 years), gender, and index year. The patients in both groups were free of cancer before and on the index date. The same inclusion and exclusion criteria were used for the non-NTS group and the NTS group.

\section{Definition of variables}

We extracted clinical information from the dataset of records from 1998 to 2000. Underlying general conditions and relevant covariates associated with gastrointestinal malignancy consisted of hypertension (ICD-9-CM 401405), diabetes mellitus (ICD-9-CM 250), hyperlipidemia (ICD-9-CM 272), coronary artery disease (ICD-9-CM 410414), chronic kidney disease (ICD-9-CM 585), peptic ulcer disease (ICD-9-CM 531- 533), asthma (ICD-9-CM 493), chronic obstructive pulmonary disease (ICD-9-CM 491, 492, 496), Helicobacter pylori (ICD-9-CM 041.86), chronic liver diseases (ICD-9-CM 571.4), hepatitis B (ICD-9-CM 070.2, 070.3, V02.61), hepatitis C (ICD-9-CM 070.41, 070.44, 070.51, 070.54, V02.62), cholecystectomy (ICD9-OP 51.2), alcohol-related illness (ICD-9-CM 291, 303, 305, 571.0, 571.1, 571.2, 571.3, 790.3, A215, and V11.3), and gallstones (ICD-9-CM 574). We used the diagnosis of peptic ulcer disease (PUD) as a proxy of $H$. pylori infection, because about $90 \%$ of PUD patients had an H. pylori infection [18]. Gallstones and cholecystectomy have been reported in association with digestive malignancy [19]. Information on co-morbid medical disorders were obtained by tracing at least two ambulatory medical care or one inpatient record in the NHI database before the index date.

\section{Outcomes}

The main outcome of this study was the occurrence of gastric cancer (ICD-9-CM codes 151.x). A gastric cancer event was defined as a patient having a record of gastric cancer diagnosis in the CICs. All the patients were followed up until they were diagnosed with gastric cancer or dropped out of the NHI program or until the end of this study (December $31,2013)$. We also analyzed the risk for other gastrointestinal malignancies individually, such as colon (ICD-9-CM codes 153.x, 154.x), biliary tract (ICD-9-CM codes 156.x), hepatic (ICD-9-CM codes 155.x), and pancreatic (ICD9-CM codes 157.x) cancer.

\section{Negative control outcome analysis}

Negative exposure control and falsification endpoint (or negative outcome control) have been used to detect residual confounding and bias due to unobserved confounders [20].

We chose lung cancer as the falsification endpoint (ICD9-CM codes 162.x), which is not associated with NTS according to a review of current pathophysiologic mechanisms. Therefore, any association between NTS and the endpoint may indicate the presence of unmeasured confounding.

\section{Statistical analysis}

First, we compared the distribution of age, sex, and baseline comorbidities between the two groups using the Chi square test.

Second, the incidence of gastrointestinal cancer in both groups was calculated as the number of patients with gastrointestinal cancer identified during the follow-up period divided by the total time of follow-up in person-years. To present the risk of cancer between both groups, hazard ratios (HRs) and 95\% confidence intervals (CIs) were estimated using single-variable and multivariable Cox proportional hazards regression. The covariates adopted in the multivariable models included sex, age, and the above mentioned variables. Because death was a potential confounder and could bias the estimated risk of cancer, we analyzed a competing risk of death model to estimate the subhazard ratios and 95\% CIs of the incidence of gastric cancer among both groups.

Third, we plotted the cumulative incidence curves of gastric cancer for both groups by the Kaplan-Meier method, and the differences in these curves were determined by the log-rank test.

Data management and statistical analyses were implemented in SAS 9.4 version software (SAS Institute, Cary, NC, USA). The significance thresholds were set at two-sided $p<0.05$.

\section{Sensitivity analyses}

We constructed three models to validate the robustness of the study findings. Model 1 contained aHR after adjusting for age and gender. Model 2 was the main model in this study. Model 2 contained the aHR after adjusting for demographic variables and all mentioned comorbidities in the study. Model 3 used propensity score matching at a ratio of $1: 1$ to balance the baseline covariates between the two groups. 
As NTS is more severe in immunocompromised patients, we conducted another sensitivity analysis to include those with underlying condition of HIV infections and immunosuppressive therapy (Supplement Tables 1 and 2). Patients who ever used corticosteroids, hydroxychloroquine, and immunosuppressive treatment (azathioprine, cyclosporin, mycophenolate, sirolimus, tacrolimus) prior to the index hospitalization were defined as users. These additional analyses were conducted for the purpose of examining whether the finding was robust to different assumptions.

\section{Results}

Our study included 9097 hospitalized patients with NTS and 9097 matched non-NTS patients. Table 1 shows that the NTS cohort had a higher proportion of comorbidities.

Table 2 demonstrates the results of univariate and multivariate Cox proportional hazard regression analyses. The subjects with NTS had a 1.24-fold increased risk of gastrointestinal cancer (95\% CI 1.01-1.53) after adjusting for all covariates listed in Table 1.

Table 3 demonstrates the association between NTS and risks of gastrointestinal cancer in different stratifications. In the sex-subgroup analysis, females with NTS had a slightly higher risk of developing cancer (aHR, 1.29; $95 \%$ CI 0.91-1.83) than males with NTS (aHR, 1.23; 95\% CI 0.95-1.61). Males with NTS had the highest incidence rate of cancer among all sex subgroups (4.77 vs. 2.74). However, the $p$ value for interaction by sex-subgroup was not significant (0.857). In the age-subgroup analysis, compared with matched non-NTS age subgroups, those aged 50-64 years had aHR of 1.40 (95\% CI 0.93-2.10) for new-onset cancer. The $p$ value for interaction in the age-subgroup was not significant (0.309). In peptic ulcer disease-subgroup, the incidence rate of gastrointestinal malignancy among patients without history of NTS is $9.91 / 1000$ person years and the incidence rate of
Table 1 Demographic characteristics and comorbidities of patients newly diagnosed Nontyphoid salmonellosis in Taiwan during 2000-2012

\begin{tabular}{|c|c|c|c|c|}
\hline \multirow[t]{3}{*}{ Characteristics } & \multirow{3}{*}{$\begin{array}{l}\text { Total } \\
\mathrm{N}\end{array}$} & \multicolumn{2}{|c|}{ Nontyphoid salmonellosis } & \multirow[t]{3}{*}{$p$ value } \\
\hline & & $\begin{array}{l}\text { No } \\
n=9097\end{array}$ & $\begin{array}{l}\text { Yes } \\
n=9097\end{array}$ & \\
\hline & & $n(\%) /$ mean $(\mathrm{SD})$ & $n(\%) /$ mean $(\mathrm{SD})$ & \\
\hline \multicolumn{4}{|l|}{ Gender } & \multirow[t]{3}{*}{1.000} \\
\hline Female & 8868 & $4434(48.7)$ & $4434(48.7)$ & \\
\hline Male & 9326 & $4663(51.3)$ & $4663(51.3)$ & \\
\hline \multicolumn{4}{|l|}{ Age } & \multirow[t]{4}{*}{1.000} \\
\hline $20-49$ & 7838 & $3919(43.1)$ & $3919(43.1)$ & \\
\hline $50-64$ & 4142 & $2071(22.8)$ & $2071(22.8)$ & \\
\hline$\geq 65$ & 6214 & $3107(34.2)$ & $3107(34.2)$ & \\
\hline $\operatorname{Mean}(\mathrm{SD})^{\mathrm{a}}$ & & 54.1 (19.6) & $54.2(19.6)$ & 0.840 \\
\hline \multicolumn{5}{|l|}{ Baseline comorbidity } \\
\hline Hypertension & 3089 & $1125(12.4)$ & 1964 (21.6) & $<0.001$ \\
\hline Diabetes mellitus & 1866 & $595(6.5)$ & $1271(14)$ & $<0.001$ \\
\hline Hyperlipidemia & 878 & $269(3)$ & $609(6.7)$ & $<0.001$ \\
\hline Coronary artery disease & 2248 & $816(9)$ & $1432(15.7)$ & $<0.001$ \\
\hline Chronic kidney disease & 378 & $77(0.8)$ & $301(3.3)$ & $<0.001$ \\
\hline Peptic ulcer disease & 1678 & $544(6)$ & $1134(12.5)$ & $<0.001$ \\
\hline Asthma & 540 & $169(1.9)$ & $371(4.1)$ & $<0.001$ \\
\hline COPD & 872 & $252(2.8)$ & $620(6.8)$ & $<0.001$ \\
\hline Helicobacter pylori & 34 & $10(0.1)$ & $24(0.3)$ & 0.016 \\
\hline Chronic liver disease & 263 & $69(0.8)$ & $194(2.1)$ & $<0.001$ \\
\hline Hepatitis B & 233 & $48(0.5)$ & $185(2)$ & $<0.001$ \\
\hline Hepatitis C & 216 & $46(0.5)$ & $170(1.9)$ & $<0.001$ \\
\hline Cholecystectomy & 381 & $138(1.5)$ & $243(2.7)$ & $<0.001$ \\
\hline Alcohol-related illness & 253 & $42(0.5)$ & $211(2.3)$ & $<0.001$ \\
\hline Rheumatoid arthritis & 110 & $18(0.2)$ & $92(1)$ & $<0.001$ \\
\hline Gallstones & 701 & $216(2.4)$ & $485(5.3)$ & $<0.001$ \\
\hline
\end{tabular}

Chi square test

${ }^{\mathrm{a}} t$ test 
Table 2 Cox model measured hazard ratio and $95 \%$ confidence intervals of gastrointestinal cancer associated with and without NTS patients

\begin{tabular}{|c|c|c|c|c|c|}
\hline \multirow[t]{2}{*}{ Characteristics } & \multirow{2}{*}{$\begin{array}{l}\text { Event } \\
(n=367)\end{array}$} & \multicolumn{2}{|l|}{ Crude } & \multicolumn{2}{|l|}{ Adjusted } \\
\hline & & HR (95\% CI) & $p$ value & HR $(95 \% \mathrm{CI})$ & $p$ value \\
\hline \multicolumn{6}{|l|}{ NTS } \\
\hline No & 175 & Ref. & & Ref. & \\
\hline Yes & 192 & $1.25(1.02-1.53)$ & 0.034 & $1.24(1.01-1.53)$ & 0.047 \\
\hline \multicolumn{6}{|l|}{ Gender } \\
\hline Female & 132 & Ref. & & Ref. & \\
\hline Male & 235 & $1.77(1.43-2.19)$ & $<0.001$ & $1.62(1.3-2.01)$ & $<0.001$ \\
\hline \multicolumn{6}{|l|}{ Age at baseline } \\
\hline $20-49$ & 37 & Ref. & & Ref. & \\
\hline $50-64$ & 107 & $6.11(4.21-8.89)$ & $<0.001$ & $5.77(3.96-8.40)$ & $<0.001$ \\
\hline$\geq 65$ & 223 & $11.34(8.00-16.07)$ & $<0.001$ & $10.70(7.45-15.38)$ & $<0.001$ \\
\hline \multicolumn{6}{|l|}{ Comorbidities } \\
\hline Hypertension & 79 & $2.26(1.76-2.90)$ & $<0.001$ & $0.79(0.59-1.05)$ & 0.109 \\
\hline Diabetes mellitus & 72 & $3.59(2.77-4.66)$ & $<0.001$ & $1.91(1.43-2.56)$ & $<0.001$ \\
\hline Hyperlipidemia & 16 & $1.38(0.84-2.28)$ & 0.206 & $0.67(0.39-1.13)$ & 0.135 \\
\hline Coronary artery disease & 70 & $2.21(1.70-2.86)$ & $<0.001$ & $0.91(0.69-1.22)$ & 0.540 \\
\hline Chronic kidney disease & 8 & $1.96(0.97-3.94)$ & 0.061 & $1.03(0.50-2.10)$ & 0.946 \\
\hline Peptic ulcer disease & 53 & $2.56(1.91-3.43)$ & $<0.001$ & $1.10(0.80-1.52)$ & 0.560 \\
\hline Asthma & 10 & $1.36(0.73-2.56)$ & 0.334 & $0.63(0.32-1.21)$ & 0.163 \\
\hline COPD & 25 & $2.72(1.81-4.09)$ & $<0.001$ & $0.99(0.64-1.54)$ & 0.973 \\
\hline Helicobacter pylori & 1 & $3.29(0.46-23.36)$ & 0.234 & $1.41(0.19-10.27)$ & 0.732 \\
\hline Chronic liver disease & 19 & $5.11(3.22-8.11)$ & $<0.001$ & $2.35(1.43-3.86)$ & $<0.001$ \\
\hline Hepatitis B & 12 & $4.18(2.35-7.44)$ & $<0.001$ & $2.02(1.08-3.77)$ & 0.028 \\
\hline Hepatitis C & 20 & $9.32(5.92-14.66)$ & $<0.001$ & $4.74(2.90-7.72)$ & $<0.001$ \\
\hline Cholecystectomy & 19 & $3.61(2.27-5.73)$ & $<0.001$ & $2.00(1.02 \mathrm{v} 3.91)$ & 0.042 \\
\hline Alcohol-related illness & 12 & $4.22(2.37-7.50)$ & $<0.001$ & $2.86(1.54-5.28)$ & $<0.001$ \\
\hline Rheumatoid arthritis & 1 & $0.66(0.09-4.69)$ & 0.678 & $0.55(0.08-3.93)$ & 0.552 \\
\hline Gallstones & 27 & $2.90(1.96-4.30)$ & $<0.001$ & $0.74(0.41-1.32)$ & 0.310 \\
\hline
\end{tabular}

$H R$ hazard ratio, $C I$ confidence interval, $C O P D$ chronic obstructive pulmonary disease

*Adjusted HR: adjusted for age, gender and all comorbidities in Cox proportional hazards regression gastrointestinal malignancy among patients with NTS is $6.43 / 1000$ person years. NTS was associated subsequent gastrointestinal cancer in general, and there were no statistically significant subgroup association between NTS and gastrointestinal cancer in age-, sex-, and comorbidities- stratified analyses.

Table 4 shows the incidence of specific gastrointestinal malignancy in the two groups with respect to the results of the incidence and adjusted HRs of all statistical models. In model 1, the aHR of NTS to gastric cancer was 2.11 (95\% CI 1.25-3.55); in model 2, the aHR of NTS to gastric cancer was 2.02 (95\% CI 1.18-3.45) after adjusting for gender, age, and comorbidities at baseline; in model 3, with propensity score matching (Supplement Table 3; in both groups, each comorbiditiy with an SMD $<0.1$ indicates negligible difference), the aHR of NTS to gastric cancer was 2.82 after adjusting for gender, age, and comorbidities.

Table 5 displays the analysis stratified by the follow-up time, the NTS group had a higher risk of gastrointestinal cancer occurrence within 3 years after the index date $(\mathrm{aHR}=1.44 ; 95 \%$ CI $1.04-1.99)$.

Death was considered a competing factor, because elderly patients with NTS had a higher in-hospital mortality rate [21]. After adjusting for the covariates and considering death as a competing event, subjects with NTS had a modestly higher risk of gastric cancer than controls (adjusted subhazard ratio, 1.83; 95\% CI 1.06-3.14, in Table 6).

Another sensitivity analysis, taking HIV infections and immunosuppressive therapy into consideration, compared to those without NTS, the NTS group remains a higher risk of subsequent of gastrointestinal cancer $(\mathrm{aHR}=1.45$, 95\% CI 1.02-2.07, $p=0.04$ ) (Supplement Tables 1 and 2).

The Kaplan-Meier curve reveals that the cumulative incidence of gastric cancer was modestly increased in the NTS group compared with the non-NTS group (log-rank test, $p$ value $=0.0334$, Fig. 1 ) . 


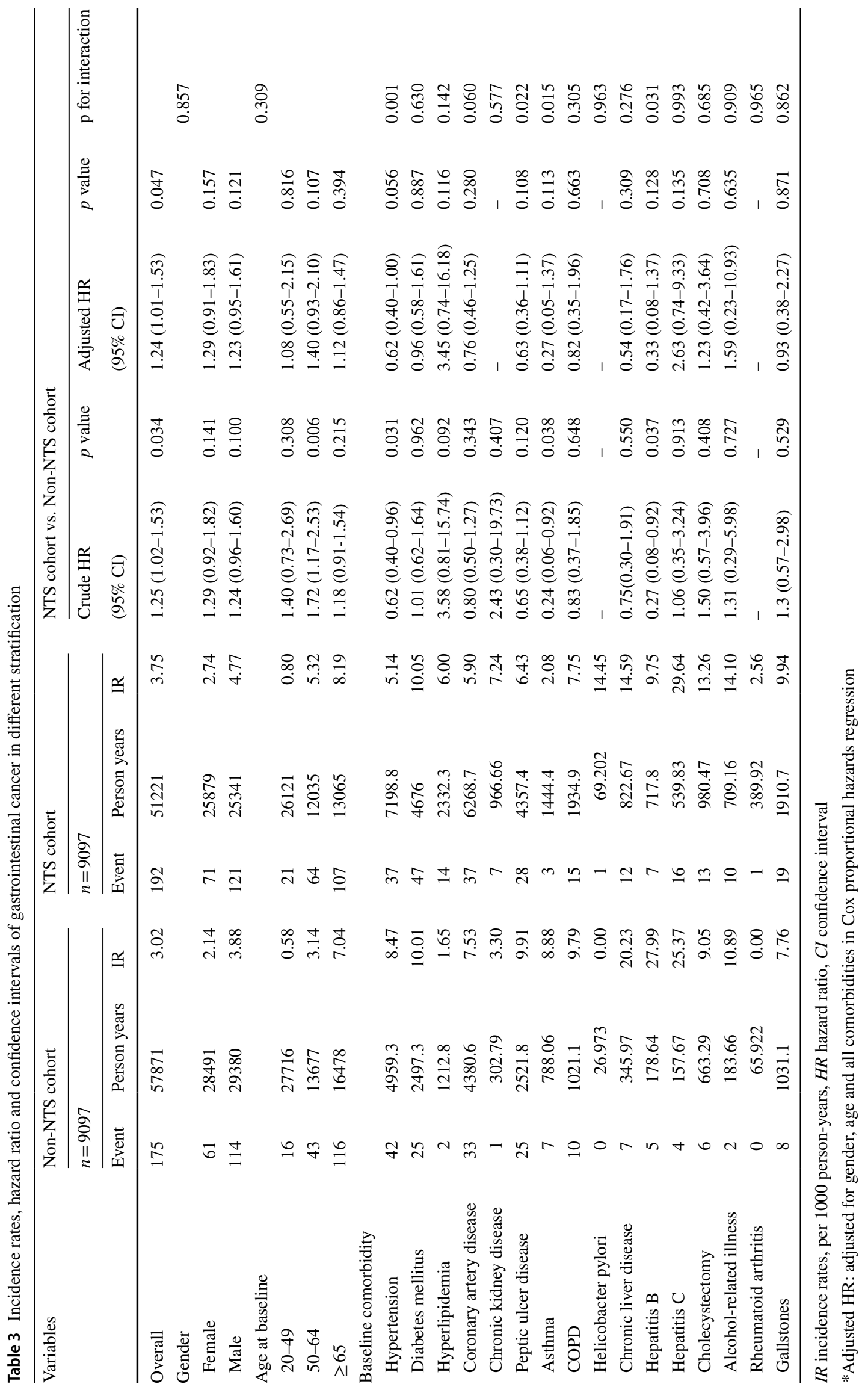


Table 4 Incidence and hazard ratio of different type of gastrointestinal cancer

\begin{tabular}{|c|c|c|c|c|c|c|c|c|c|c|}
\hline \multirow[t]{3}{*}{ Cancer } & \multicolumn{4}{|c|}{ Nontyphoid salmonellosis } & \multirow{2}{*}{\multicolumn{2}{|c|}{ Model 1}} & \multirow{2}{*}{\multicolumn{2}{|c|}{ Model 2}} & \multirow{2}{*}{\multicolumn{2}{|c|}{ Model 3}} \\
\hline & \multicolumn{2}{|l|}{ No } & \multicolumn{2}{|l|}{ Yes } & & & & & & \\
\hline & Event & IR & Event & IR & aHR $(95 \% \mathrm{CI})$ & $p$ value & aHR $(95 \% \mathrm{CI})$ & $p$ value & aHR $(95 \% \mathrm{CI})$ & $p$ value \\
\hline Colorectal & 67 & 1.16 & 55 & 1.07 & $1.03(0.72-1.47)$ & 0.884 & $1.04(0.72-1.50)$ & 0.844 & $1.50(0.73-3.11)$ & 0.274 \\
\hline Stomach & 23 & 0.40 & 37 & 0.72 & $2.11(1.25-3.55)$ & 0.005 & $2.02(1.18-3.45)$ & 0.010 & $2.82(1.00-8.11)$ & 0.050 \\
\hline Biliary tract & 6 & 0.10 & 11 & 0.21 & $2.23(0.83-6.05)$ & 0.114 & $1.79(0.65-4.97)$ & 0.264 & $1.66(0.29-9.41)$ & 0.569 \\
\hline Liver & 64 & 1.11 & 70 & 1.37 & $1.35(0.96-1.90)$ & 0.083 & $1.03(0.72-1.47)$ & 0.877 & $1.01(0.47-2.18)$ & 0.980 \\
\hline Pancreas & 9 & 0.16 & 7 & 0.14 & $0.92(0.34-2.49)$ & 0.876 & $0.81(0.29-2.30)$ & 0.694 & $0.72(0.16-3.29)$ & 0.668 \\
\hline
\end{tabular}

PY: person-years; IR: incidence rate, per 1000-person years; HR: hazard ratio; CI: confidence interval

Model 1 Frequency matched 2 groups, excluded outcome $<6$ months of index date; and adjusted by age and gender in Cox proportional hazards regression

Model 2 Frequency matched 2 groups, excluded outcome $<6$ months of index date; and adjusted by age, gender and all mentioned comorbidities in Cox proportional hazards regression

Model 3 Propensity score matched 2 groups, excluded outcome $<1$ year of index date; adjusted by age, gender and all mentioned comorbidities in Cox proportional hazards regression with a robust sandwich covariance estimator

Table 5 Incidence rates, hazard ratio and confidence intervals of gastrointestinal cancer in different stratification of follow-up years

\begin{tabular}{|c|c|c|c|c|c|c|c|c|c|c|}
\hline \multirow[t]{3}{*}{ Variables } & \multirow{2}{*}{\multicolumn{3}{|c|}{$\begin{array}{l}\text { Non-NTS cohort } \\
n=9097\end{array}$}} & \multirow{2}{*}{\multicolumn{3}{|c|}{$\begin{array}{l}\text { NTS cohort } \\
n=9097\end{array}$}} & \multicolumn{4}{|c|}{ NTS cohort vs. Non-NTS cohort } \\
\hline & & & & & & & \multirow{2}{*}{$\begin{array}{l}\text { Crude HR } \\
(95 \% \mathrm{CI})\end{array}$} & \multirow[t]{2}{*}{$p$ value } & \multirow{2}{*}{$\begin{array}{l}\text { Adjusted HR } \\
(95 \% \mathrm{CI})\end{array}$} & \multirow[t]{2}{*}{$p$ value } \\
\hline & Event & Person years & IR & Event & Person years & IR & & & & \\
\hline \multicolumn{11}{|c|}{ Follow-up years } \\
\hline$<3$ & 69 & 24855 & 2.78 & 89 & 22844 & 3.90 & $1.42(1.04-1.94)$ & 0.029 & $1.44(1.04-1.99)$ & 0.030 \\
\hline$\geq 3$ & 106 & 33015 & 3.21 & 103 & 28377 & 3.63 & $1.13(0.87-1.49)$ & 0.363 & $1.10(0.83-1.46)$ & 0.496 \\
\hline
\end{tabular}

$I R$ incidence rates, per 1000 person-years, $H R$ hazard ratio, $C I$ confidence interval

Table 6 NTS group to Non-NTS group subhazard ratio (SHR) of gastric cancer estimated through competing-risks regression

\begin{tabular}{llll}
\hline Variable & NTS & \multirow{2}{*}{$p$ value } \\
\cline { 2 - 3 } & No & Yes & \\
\hline Cancer & & & \\
Crude SHR (95\% CI) & 1.00 (Ref.) & $1.72(1.02-2.91)$ & 0.042 \\
Adjusted SHR ${ }^{\dagger}$ (95\% CI) & 1.00 (Ref.) & $1.83(1.06-3.14)$ & 0.029 \\
\hline
\end{tabular}

Crude SHR: relative subhazard ratio

Adjusted $\mathrm{SHR}^{\dagger}$ : multivariable analysis including all factors in the univariable cox model

\section{Negative control outcome}

The result of negative control analysis showed a non-significant association between NTS and lung cancer (aHR, $1.11 ; 95 \%$ CI 0.74-1.67). Unmeasured confounding was not present from this point of view. Using negative controls, still we can not completely deal with unmeasured confounding in our study, but we adopted this measurement trying to address the potential magnitude of uncontrolled confounder is minimal.

\section{Discussion}

This study revealed an increased risk of gastric cancer in the NTS group compared with the age-, sex-, and index date-matched non-NTS group. Furthermore, after crossvalidation by sensitivity analyses and negative controls, our findings indicate that nontyphoid Salmonella infection may be an independent risk factor for gastric cancer. We re-analyzed the study by propensity score matching to minimize the difference in comorbidity distribution in both groups. The results were consistent.

In the traditional concept of gastric cancer formation, the disease encompasses both a chronic inflammatory state and direct genotoxic effects on gastric mucosa [22]. Although $H$. pylori is a well-known risk factor, merely $3 \%$ of $H$. pylori-infected people develop gastric cancer [8]. H. pylori 


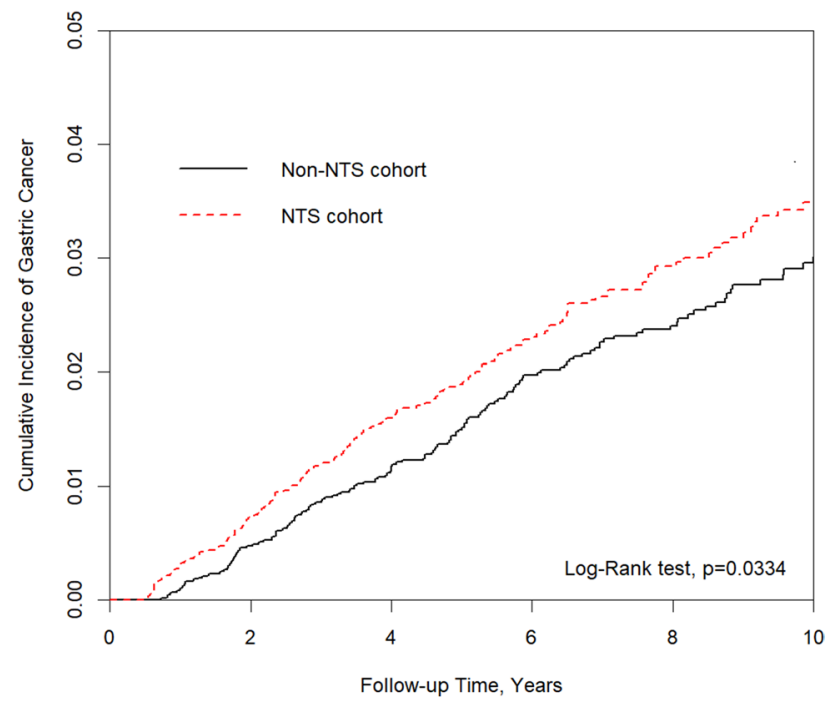

Fig. 1 Cumulative curve of gastric cancer for the NTS group was significantly higher than that of the comparison groups (log-rank test, $\mathrm{P}=0.0334)$

eradication alone does not eliminate gastric cancer, indicating that a complicated underlying process and pathogens other than H. pylori may be involved [23]. In recent studies, Salmonella enterica has been associated with the development of gallbladder cancer and colon cancer [7, 24]. On the contrary, attenuated strains have been evaluated as adjuvants in the treatment of cancer for their affinity for tumor tissue [25]. Our study, based on a real-world nationwide dataset, revealed that patients with histories of NTS hospitalization had a higher risk of gastric cancer.

The mechanisms for the increased risks of gastric cancers in the NTS population have not yet been elucidated. Notwithstanding this, NTS is clearly versatile in at least two aspects: first, it causes a greater disturbance in the microbiome than a bout of gastroenteritis [26-29], and second, it reprograms the characteristics of macrophages [15]. Because of the rapid evolution of sequencing technology, we are now more familiar with highly complex microbiota in the gut and stomach. Even with the acidity of the gastric environment, the gastrointestinal lumen is rich in microbiota. Microbial dysbiosis, an inner environmental issue, is also associated with gastric carcinogenesis [30]. It is now known that Actinobacteria, Firmicutes, Bacteroidetes, and Proteobacteria are the predominant phyla in the human stomach [31]. The growth and decline of gut microbiota are in a dynamic balance, which can be affected by dietary habits, the use of drugs, e.g., antibiotics and proton pump inhibitors, and opportunistic pathogens, such as NTS, all of which may extensively disturb microbiome homeostasis [32]. There is supporting evidence that generalized dysbiosis may contribute to carcinomatosis [33]. Another potential mechanism may be the interactions between immune pathways involved in infectious agents and cancer cells [3]. Macrophages, arising from circulating monocytes, are notable in the human immune system and widely distributed in the mucosal layers of the gastrointestinal lumen. There are two main types of macrophages-M1 and M2 phenotypes-depending on microenvironmental cues. Cells with the M1 phenotype play roles in anti-tumor immunity [34, 35], and M2 cells influence wound healing, pro-tumorigenic properties, and anti-inflammatory agents by immunosuppression [36]. Furthermore, M2 cells have an impact on tumor proliferation and invasion [37]. Tumor-associated macrophages (TAMs) are referred to as M2-like, maturing in the tumor-tissue environment after leaving the blood circulation as monocytes. One recent study discovered that the transcription factor c-Maf was highly expressed in human immunosuppressive TAMs and polarized M2 macrophages [38]. Another study revealed that c-Maf was markedly increased in Salmonellainfected macrophages [39], and c-Maf was also involved in gut homeostasis by regulating $\mathrm{T}_{\text {reg }}$ and $\mathrm{T}_{\mathrm{H}} 17$ cells [40]. Furthermore, Salmonellae can escape from immunological surveillance [17] and replicate within macrophages, dendritic cells, and neutrophils and become persisters, which can reprogram the macrophage [15]. Researchers once found Salmonella typhimurium entering macrophages, and some of them could induce the expression of the Salmonella pathogenicity island 2 type III secretion system to inhibit M1 and drive M2 macrophage polarization [15]. The effect of NTScontaining macrophages is supposed to be M2-like, which suppresses human anti-tumor function and causes gastric cancer development. Furthermore, one recent study revealed that the use of oral PPIs was associated with the occurrence of NTS [41]. It is likely that more bacteria in NTS survive in a relatively high $\mathrm{pH}$ environment. However, the detailed interactions between NTS and neoplastic changes in gastric epithelial cells need further investigation.

To the best of our knowledge, based on a literature review, this is a novel retrospective cohort study that used nationwide big data to evaluate the long-term risk of gastric cancer among patients with NTS. A particular strength of this study is its population-based study design, which allowed us to identify nearly all patients hospitalized with a principal diagnosis of NTS and cancer in Taiwan and thus minimize referral bias, as all medical care is covered by the national insurance system. The NHI program is universal and mandatory in Taiwan, and NHI beneficiaries are assigned personal identification numbers that enable them to be traced throughout the follow-up period. Moreover, the cancer diagnoses in our study were verified by the Registry for Catastrophic Illness Patient Database, and the certification is linked to the financial entity afterward for each patient. The study used 
sensitivity analyses and negative control analyses to validate the main finding.

Some limitations of our study should be noted. First, cancer was not identified by medical records but defined by ICD-9-CM codes. Recent study showed that the sensitivity of ICD code for cancer was $91.5 \%$, with a positive predictive value of $93.6 \%$ [42]. Second, the inclusion of NTS cases that were dependent on ICD-9-CM codes may be less accurate than those in a prospective clinical setting. For coding accuracy, the study enrolled patients hospitalized with the principal ICD-9-CM code of NTS [41]. The people with non-hospitalized NTS are beyond the scope of the study, because they may have a low bacterial load of nontyphoid Salmonella. Besides, NTS patients with milder symptoms do not submit claims for medical services and those may have been misclassified into the comparison group. If NTS is associated causally with gastric cancer, patient-misclassification would bias the HRs estimation toward the null. Therefore, the results are reliable. Third, information on some potential risk factors, such as tobacco and alcohol consumption, environmental exposure, and family history of malignancy, was not available in NHIRD. Nonetheless, some smoking-related health consequences were partly reflected in the presentation of comorbidities such as hypertension, chronic obstructive pulmonary disease, and coronary heart disease, which were included in the analysis. Laboratory report including CagA-positive H. pylori infection or score of inflammatory cell infiltration cannot be provided in the NHIRD and this is a major limitation in the study. Fourth, Salmonella has its specific tropism to tumor microenvironment, so we used the lag-time approach excluding cancer detection date within 1 year after the NTS diagnosis date to ameliorate protopathic bias. Finally, this study can not determine the direction of causality, but through a cohort study using insurance data with a meticulous design, including propensity score matching for measurable confounders and negative controls for unmeasured confounders, is a suitable alternative to a dedicated prospective one to analyze the risk factors [43-45].

\section{Conclusion}

Patients with histories of NTS have a modestly increased risk of developing gastric cancer. Our findings are helpful in improving gastric cancer risk evaluation and prevention. Whether antibiotic therapy has an influence on cancer is unknown. Further comprehensive prospectives research is required to clarify the underlying biological mechanisms of these associations.
Acknowledgements The authors expressed their appreciation to the Department of Medical Education and Research and Research Center of Medical Informatics in Kaohsiung Veterans General Hospital for the comments. The authors expressed appreciation to the Department of Medical Education and Research and Research Center of Medical Informatics in Kaohsiung Municipal United Hospital for the comments.

Author contributions All the authors were involved in drafting the article or revising it and all authors approved of the final version being published.

Study conception and design: Y-MH, RC, M-CL, JC-CW. Acquisition of data: M-CL. Analysis and interpretation of data: Y-MH, RC, M-CL, JC-CW. Writing (original draft preparation): Y-MH, RC. Writing (review and editing): Y-MH, JC-CW

Funding This study is supported in part by Taiwan Ministry of Health and Welfare Clinical Trial Center (MOHW109-TDU-B-212-114004), MOST Clinical Trial Consortium for Stroke (MOST Clinical Trial Consortium for Stroke (MOST 109-2321-B-039-002)), Tseng-Lien Lin Foundation, Taichung, Taiwan.

\section{Compliance with ethical standards}

Conflict of interest :All of the authors have declared no competing interest.

\section{References}

1. Ohshima H, Bartsch H. Chronic infections and inflammatory processes as cancer risk factors: possible role of nitric oxide in carcinogenesis. Mutat Res. 1994;305:253-64.

2. Pisani P, Parkin DM, Munoz N, Ferlay J. Cancer and infection: estimates of the attributable fraction in 1990. Cancer Epidemiol Biomarkers Prev. 1997;6:387-400.

3. Jacqueline C, Tasiemski A, Sorci G, Ujvari B, Maachi F, Misse $\mathrm{D}$, et al. Infections and cancer: the "fifty shades of immunity" hypothesis. BMC Cancer. 2017;17:257.

4. Gagnaire A, Nadel B, Raoult D, Neefjes J, Gorvel JP. Collateral damage: insights into bacterial mechanisms that predispose host cells to cancer. Nat Rev Microbiol. 2017;15:109-28.

5. Boccellato F, Meyer TF. Bacteria moving into focus of human cancer. Cell Host Microbe. 2015;17:728-30.

6. Berger H, Marques MS, Zietlow R, Meyer TF, Machado JC, Figueiredo C. Gastric cancer pathogenesis. Helicobacter. 2016;21(Suppl 1):34-8.

7. Scanu T, Spaapen RM, Bakker JM, Pratap CB, Wu LE, Hofland I, et al. Salmonella manipulation of host signaling pathways provokes cellular transformation associated with gallbladder carcinoma. Cell Host Microb. 2015;17:763-74.

8. Peek RM Jr, Crabtree JE. Helicobacter infection and gastric neoplasia. J Pathol. 2006;208:233-48.

9. Chang JS, Tsai CR, Chen LT, Shan YS. Investigating the Association between periodontal disease and risk of pancreatic cancer. Pancreas. 2016;45:134-41.

10. Jacob JA. Study links periodontal disease bacteria to pancreatic cancer risk. JAMA. 2016;315:2653-4.

11. Li P, Shu Y, Gu Y. The potential role of bacteria in pancreatic cancer: a systematic review. Carcinogenesis. 2020;41:397-404.

12. Mughini-Gras L, Schaapveld M, Kramers J, Mooij S, NeefjesBorst EA, Pelt WV, et al. Increased colon cancer risk after severe Salmonella infection. PLoS ONE. 2018;13:e189721. 
13. Lu R, Wu S, Zhang YG, Xia Y, Liu X, Zheng Y, et al. Enteric bacterial protein AvrA promotes colonic tumorigenesis and activates colonic beta-catenin signaling pathway. Oncogenesis. 2014;3:e105.

14. Voetsch AC, Van Gilder TJ, Angulo FJ, Farley MM, Shallow $\mathrm{S}$, Marcus R, et al. FoodNet estimate of the burden of illness caused by nontyphoidal Salmonella infections in the United States. Clin Infect Dis. 2004;38(Suppl 3):S127-34.

15. Darwin KH, Miller VL. Molecular basis of the interaction of Salmonella with the intestinal mucosa. Clin Microbiol Rev. 1999; 12:405-28.

16. Stapels DAC, Hill PWS, Westermann AJ, Fisher RA, Thurston TL, Saliba AE, et al. Salmonella persisters undermine host immune defenses during antibiotic treatment. Science. 2018;362:1156-60.

17. Meng C, Bai C, Brown TD, Hood LE, Tian Q. Human Gut Microbiota and Gastrointestinal Cancer. Genomics Proteomics Bioinformatics. 2018;16:33-49.

18. Kurata JH, Nogawa AN. Meta-analysis of risk factors for peptic ulcer. Nonsteroidal antiinflammatory drugs, Helicobacter pylori, and smoking. J Clin Gastroenterol. 1997;24:2-17.

19. Nogueira L, Freedman ND, Engels EA, Warren JL, Castro F, Koshiol J. Gallstones, cholecystectomy, and risk of digestive system cancers. Am J Epidemiol. 2014;179:731-9.

20. Norgaard M, Ehrenstein V, Vandenbroucke JP. Confounding in observational studies based on large health care databases: problems and potential solutions - a primer for the clinician. Clin Epidemiol. 2017;9:185-93.

21. Chen PL, Li CY, Hsieh TH, Chang CM, Lee HC, Lee NY, et al. Epidemiology, disease spectrum and economic burden of nontyphoidal Salmonella infections in Taiwan, 2006-2008. Epidemiol Infect. 2012;140:2256-63.

22. Helmink BA, Khan MAW, Hermann A, Gopalakrishnan V, Wargo JA. The microbiome, cancer, and cancer therapy. Nat Med. 2019;25:377-88.

23. Coker OO, Dai Z, Nie Y, Zhao G, Cao L, Nakatsu G, et al. Mucosal microbiome dysbiosis in gastric carcinogenesis. Gut. 2018;67:1024-32.

24. Liu X, Jiang S, Piao L, Yuan F. Radiotherapy combined with an engineered Salmonella typhimurium inhibits tumor growth in a mouse model of colon cancer. Exp Anim. 2016;65:413-8.

25. Chavez-Navarro H, Hernandez-Cueto DD, Vilchis-Estrada A, Bermudez-Pulido DC, Antonio-Andres G. Luria-Perez R [Salmonella enterica: an ally in the therapy of cancer]. Bol Med Hosp Infant Mex. 2015;72:15-25.

26. Santos RL. Pathobiology of salmonella, intestinal microbiota, and the host innate immune response. Front Immunol. 2014;5:252.

27. Stelter C, Kappeli R, Konig C, Krah A, Hardt WD, Stecher B, et al. Salmonella-induced mucosal lectin RegIIIbeta kills competing gut microbiota. PLoS ONE. 2011;6:e20749.

28. Schultz BM, Paduro CA, Salazar GA, Salazar-Echegarai FJ, Sebastian VP, Riedel CA, et al. A potential role of salmonella infection in the onset of inflammatory bowel diseases. Front Immunol. 2017;8:191.

29. Schultz BM, Salazar GA, Paduro CA, Pardo-Roa C, Pizarro DP, Salazar-Echegarai FJ, et al. Persistent Salmonella enterica serovar typhimurium infection increases the susceptibility of mice to develop intestinal inflammation. Front Immunol. 2018;9:1166.

30. Aviles-Jimenez F, Vazquez-Jimenez F, Medrano-Guzman R, Mantilla A, Torres J. Stomach microbiota composition varies between patients with non-atrophic gastritis and patients with intestinal type of gastric cancer. Sci Rep. 2014;4:4202.

31. Eckburg PB, Bik EM, Bernstein CN, Purdom E, Dethlefsen L, Sargent M, et al. Diversity of the human intestinal microbial flora. Science. 2005;308:1635-8.

32. Arguello H, Estelle J, Leonard FC, Crispie F, Cotter PD, O'Sullivan $\mathrm{O}$, et al. Influence of the intestinal microbiota on colonization resistance to salmonella and the shedding pattern of naturally exposed pigs. MSystems. 2019;4(2):00021.

33. Tsilimigras MC, Fodor A, Jobin C. Carcinogenesis and therapeutics: the microbiota perspective. Nat Microbiol. 2017;2:17008.

34. Chanmee T, Ontong P, Konno K, Itano N. Tumor-associated macrophages as major players in the tumor microenvironment. Cancers (Basel). 2014;6:1670-90.

35. Traves PG, Luque A, Hortelano S. Macrophages, inflammation, and tumor suppressors: ARF, a new player in the game. Mediators Inflamm. 2012;2012:568783.

36. Paolino M, Penninger JM. The role of TAM family receptors in immune cell function: implications for cancer therapy. Cancers (Basel). 2016;8(10):97.

37. Dwyer AR, Greenland EL, Pixley FJ. Promotion of tumor invasion by tumor-associated macrophages: the role of csf-1-activated phosphatidylinositol 3 kinase and src family kinase motility signaling. Cancers (Basel). 2017;9(6):68.

38. Kikuchi K, Iida M, Ikeda N, Moriyama S, Hamada M, Takahashi $\mathrm{S}$, et al. Macrophages switch their phenotype by regulating maf expression during different phases of inflammation. J Immunol. 2018;201:635-51.

39. Elhofy A, Marriott I, Bost KL. Salmonella infection does not increase expression and activity of the high affinity IL-12 receptor. J Immunol. 2000;165:3324-32.

40. Imbratta $\mathrm{C}$, Leblond MM, Bouzourene $\mathrm{H}$, Speiser DE, Velin D, Verdeil G. Maf deficiency in T cells dysregulates Treg-TH17 balance leading to spontaneous colitis. Sci Rep. 2019;9:6135.

41. Wu HH, Chen YT, Shih CJ, Lee YT, Kuo SC, Chen TL. Association between recent use of proton pump inhibitors and nontyphoid salmonellosis: a nested case-control study. Clin Infect Dis. 2014;59:1554-8.

42. Kao WH, Hong JH, See LC, Yu HP, Hsu JT, Chou IJ, et al. Validity of cancer diagnosis in the National Health Insurance database compared with the linked National Cancer Registry in Taiwan. Pharmacoepidemiol Drug Saf. 2018;27:1060-6.

43. Hsing AW, Ioannidis JP. Nationwide population science: lessons from the Taiwan National Health Insurance Research Database. JAMA Intern Med. 2015;175:1527-9.

44. Lin JN, Lin CL, Lin MC, Lai CH, Lin HH, Yang CH, et al. Risk of leukaemia in children infected with enterovirus: a nationwide, retrospective, population-based, Taiwanese-registry, cohort study. Lancet Oncol. 2015;16:1335-43.

45. Chen ML, Kao WM, Huang JY, Hung YM, Wei JC. Human papillomavirus infection associated with increased risk of new-onset psoriasis: a nationwide population-based cohort study. Int J Epidemiol. 2020;49:786-97.

Publisher's Note Springer Nature remains neutral with regard to jurisdictional claims in published maps and institutional affiliations. 